\title{
Major complications from radiotherapy following treatment for atypical meningiomas
}

\author{
Troy Dawley, DO, Zaker Rana, MD, ${ }^{2}$ Hussam Abou-AI-Shaar, MD, ${ }^{3}$ Anuj Goenka, MD, ${ }^{2}$ and \\ Michael Schulder, MD³
}

'Section of Neurosurgery, Department of Surgery, Ascension Providence Hospitals, Michigan State University, College of Human Medicine, Southfield, Michigan; and Departments of ${ }^{2}$ Radiation Oncology and ${ }^{3}$ Neurosurgery, Zucker School of Medicine at Hofstra/Northwell, Lake Success, New York

OBJECTIVE Complications from radiotherapy (RT), in a primary or adjuvant setting, have overall been described as uncommon, with few detailed descriptions of major complications. The authors present two cases involving significant complications and their management in their review of patients undergoing RT for treatment of atypical meningioma.

METHODS The authors conducted a retrospective review of all patients with pathologically confirmed atypical meningioma (WHO grade II) treated with primary or adjuvant RT from February 2011 through February 2019. They identified two patients with long-term, grade 3 toxicity. The cases of these patients are described in detail.

RESULTS Two patients had major complications associated with postoperative RT. Patients 1 and 2 both were treated with postoperative RT for pathologically confirmed atypical meningioma. Patient 1 experienced worsening behavioral changes, cognitive decline, and hydrocephalus following treatment. This required cerebrospinal fluid diversion. Patient 2 developed radiation necrosis with mass effect and cognitive decline. Neither patient returned to his/her initial post-RT status after steroid therapy, and each remained in need of supportive care. Both patients remained free of tumor progression at 52 and 38 months following treatment.

CONCLUSIONS The postoperative management of patients with atypical meningioma continues to be defined, with questions remaining regarding timing of RT, dose, target delineation, and fractionation. Both of the patients in this study received fractionated $\mathrm{RT}$, which included a greater volume of normal brain than more focal treatment options such as would be required by stereotactic radiosurgery (SRS). Further research is needed to compare SRS and fractionated RT for the management of patients with grade II meningiomas. The more focused nature of SRS may make this a preferred option in certain cases of focal recurrence.

https://thejns.org/doi/abs/10.3171/2019.3.FOCUS1930

KEYWORDS radiotherapy; atypical meningioma; radiation necrosis; complication

$\mathrm{M}$ ENINGIOMAS are the most common intracranial tumors. They include WHO grade I (81.1\%), "atypical" WHO grade II (16.9\%), and anaplastic $(1.7 \%)$ or WHO grade III tumors..$^{28}$ While grade I and II tumors have a female predominance, anaplastic (malignant) meningiomas do not. ${ }^{1}$ The 10 -year overall survival (OS) for patients with meningiomas is age dependent and ranges from $57 \%$ to $77 \% .{ }^{8}$ However, patients with grade II and III tumors have significant recurrence rates at 5 years $-50 \%$ and $90 \%$, respectively. ${ }^{8}$ Despite current advances, the 10 - year survival rates for these patients are $53 \%$ and $0 \%$, respectively. ${ }^{8}$

Treatment options for patients with atypical meningiomas include observation, resection, and radiotherapy (RT), in the form of stereotactic radiosurgery (SRS), fractionated radiotherapy (FRT), or a combination of the above. Considerations for treatment options include local mass effect, neurological symptoms, and perilesional edema. Following gross-total resection (GTR), observation or adjuvant RT is recommended and is the topic of the ongoing prospective

ABBREVIATIONS ADL = activities of daily living; CTV = clinical tumor volume; FRT = fractionated radiotherapy; GTR = gross-total resection; GTV = gross tumor volume; PTV = planning tumor volume; OS = overall survival; RT = radiotherapy; SRS = stereotactic radiosurgery; STR = subtotal resection.

SUBMITTED February 1, 2019. ACCEPTED March 21, 2019.

INCLUDE WHEN CITING DOI: 10.3171/2019.3.FOCUS1930. 
trial NRG-BN003, in which patients are treated with adjuvant RT to a dose of $54 \mathrm{~Gy} .{ }^{16}$ Following subtotal or partial resection, the standard of care is to recommend adjuvant RT, which has been shown to improve local control and overall survival. 11,31,32 Current National Comprehensive Cancer Network guidelines recommend a margin between 1 and $2 \mathrm{~cm}$ for a dose of 54-60 Gy. ${ }^{27}$ However, there are multiple retrospective studies that have looked at utilizing SRS rather than FRT and have demonstrated good local control and an excellent toxicity profile., $, 13,17,35$

Side effects from RT include symptomatic edema that can result in headaches, dizziness, seizures, and cognitive decline, as well as long-term risks of neurocognitive deficits, cranial nerve deficits, and secondary malignancy., 4 Major complications from FRT are uncommon. In this article, we highlight two patients with atypical meningioma treated with adjuvant RT who had poor clinical outcomes despite disease control.

\section{Methods}

In our single institution, we identified two patients with long-term grade 3 toxicity after adjuvant RT for pathologically confirmed atypical (WHO grade II) meningiomas. The clinical records for these two patients were reviewed by the neurosurgery and radiation oncology teams retrospectively. Further analysis of all patients who underwent treatment of WHO grade II meningiomas with adjuvant or primary RT is currently underway at our institution.

\section{Results}

During the study period from February 2011 through February 2019, a total of 91 patients underwent primary or adjuvant RT for intracranial meningiomas (Table 1). Two patients had major complications associated with postoperative RT in our review. Their cases are reviewed and described below.

\section{Illustrative Cases}

\section{Patient 1}

\section{Clinical Presentation and Operation}

A 61-year-old, right-handed man with a history of diabetes mellitus presented with 1 week of headaches and confusion. Neurological examination was positive for disorientation to place and time. The rest of his examination was unremarkable. MRI revealed a $3.5 \times 4.8 \times 3.6-\mathrm{cm}$ left frontal lesion with adjacent dural enhancement, causing mass effect on the left frontal horn (Fig. 1). The patient was started on steroids and began to experience some improvement in clinical symptoms. At the time of surgery our assessment was that a GTR (Simpson grade I) ${ }^{34}$ of his lesion was achieved via a left frontal craniotomy.

\section{Pathological Findings}

Histopathological examination of the lesion revealed an atypical meningioma, WHO grade II, with moderate to focally high cellularity. Immunohistochemistry showed $10 \%$ cells with progesterone immunoreactivity and a $\mathrm{Ki}-67$ index of $25 \%$ (Fig. 2). Immunoreactivity for p53 was found in $3 \%$ of tumor cells. A high mitotic index was found to be 10 mitoses/10 hpf.
TABLE 1. Summary of baseline characteristics in 91 patients who underwent primary or adjuvant RT to treat an intracranial meningioma

\begin{tabular}{|c|c|}
\hline Characteristic & Value \\
\hline \multicolumn{2}{|l|}{ Age at treatment, yrs } \\
\hline Median & 60 \\
\hline Range & $38-85$ \\
\hline \multicolumn{2}{|l|}{ Follow-up, mos } \\
\hline Median & 39 \\
\hline Range & $3-98$ \\
\hline \multicolumn{2}{|l|}{ Race, no. } \\
\hline White & 32 \\
\hline African American & 22 \\
\hline Hispanic & 19 \\
\hline Asian/Pacific Islander & 18 \\
\hline \multicolumn{2}{|l|}{ Sex, no. } \\
\hline Male & 41 \\
\hline Female & 50 \\
\hline \multicolumn{2}{|l|}{ Tumor grade, no. } \\
\hline No biopsy & 15 \\
\hline Grade 1 & 23 \\
\hline Grade 2 & 49 \\
\hline Grade 3 & 4 \\
\hline \multicolumn{2}{|l|}{ Location, no. } \\
\hline Anterior fossa & 13 \\
\hline Middle fossa & 14 \\
\hline Posterior fossa & 18 \\
\hline Convexity & 24 \\
\hline Parasagittal & 13 \\
\hline Temporal & 6 \\
\hline Other & 3 \\
\hline \multicolumn{2}{|c|}{ Worst overall toxicity, no. (\%) } \\
\hline None recorded & $20(22 \%)$ \\
\hline Grade 1 & $50(55 \%)$ \\
\hline Grade 2 & $19(21 \%)$ \\
\hline Grade 3 & $2(2 \%)$ \\
\hline Grade 4+ & 0 \\
\hline Total & 91 \\
\hline
\end{tabular}

\section{Postoperative Course}

The patient tolerated the procedure well with no complications. He was discharged to home on postoperative day 5. An MR image 1 month following surgery revealed nodular enhancement along the left frontal surgical margin, along with persistent vasogenic edema and right subfalcine herniation. Symptomatically, the patient was noted to have continued memory and speech difficulties. He began a course of FRT 9 weeks after surgery with a total treatment dose of $60 \mathrm{~Gy}(2 \mathrm{~Gy} \times 30$ fractions, Fig. 3). Fractionated treatment was offered due to the size of the cavity, the concern of residual disease on postoperative imaging, and the pathology findings.

Three weeks into his radiation treatment, the patient required craniectomy and evacuation of a left frontal epi- 


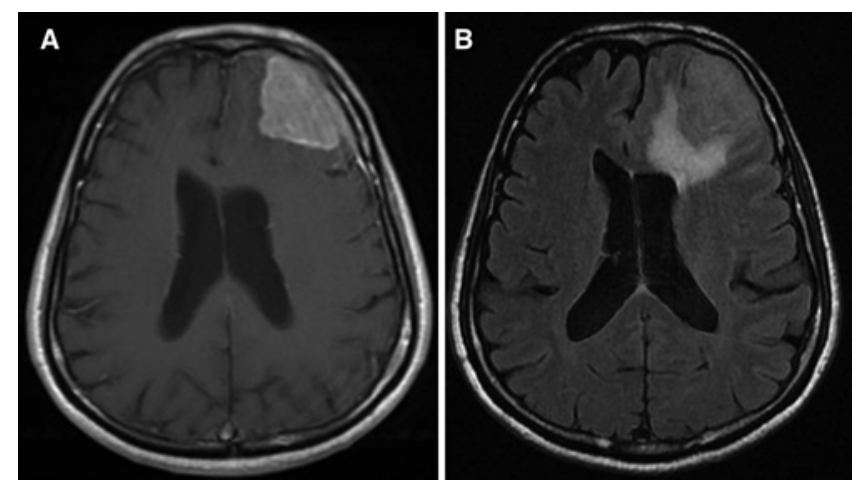

FIG. 1. Patient 1. Preoperative contrast-enhanced T1-weighted (A) and T2-weighted FLAIR (B) MR images demonstrating a left frontal lesion with adjacent dural enhancement, causing mass effect on the left frontal horn and surrounding vasogenic edema.

dural abscess, along with a titanium mesh cranioplasty. RT was completed to the planned dose of $60 \mathrm{~Gy}$ after the incision healed, with a 2-week interruption.

Surveillance imaging 4 months later noted resolution of his abscess and decreased edema. However, there was residual T2/FLAIR signal abnormality along the left frontal horn, as well as mild ex vacuo dilatation. The patient was able to return to work as a warehouse supervisor, although his effectiveness was limited by emotional lability and disinhibition.

Over the next 18 months, surveillance imaging showed a further increase in the size of the enhancement along the frontal horn and an increased degree of the ex vacuo enlargement of the frontal horn of the left lateral ventricle (Fig. 4A and B), with no evidence of tumor recurrence. The patient complained of progressive fatigue, difficulty concentrating, and that he could no longer perform his duties at work. On examination, he was speaking less and lacked of insight into his condition. He was started on a low dose of steroids without significant improvement in his symptoms.

At 24 months postoperatively, his family noted that the patient had become more erratic and aggressive. He also was more impulsive and disoriented, with difficulty walking and having frequent falls. He could no longer follow commands and had urinary and fecal incontinence. MRI revealed left frontal periventricular enhancement, with ex vacuo dilatation of the left lateral ventricle and overall enlargement of his ventricles concerning for hydrocephalus (Fig. 4C and D).

The patient underwent placement of a ventriculoperitoneal shunt and initially had neurological improvement but deteriorated over the next several weeks as his postoperative course was complicated by a small right-sided subdural hematoma from shunt overdrainage (treated by burr hole drainage and adjusting the shunt valve to a higher opening pressure). His MRI at last follow-up appointment, 52 months after surgery, demonstrated resolution of the periventricular enhancement and slightly improved T2 signal prolongation (Fig. 4E and F). He remains severely impaired with expressive and receptive aphasia, and is completely dependent on assistance for activities of daily living (ADL).

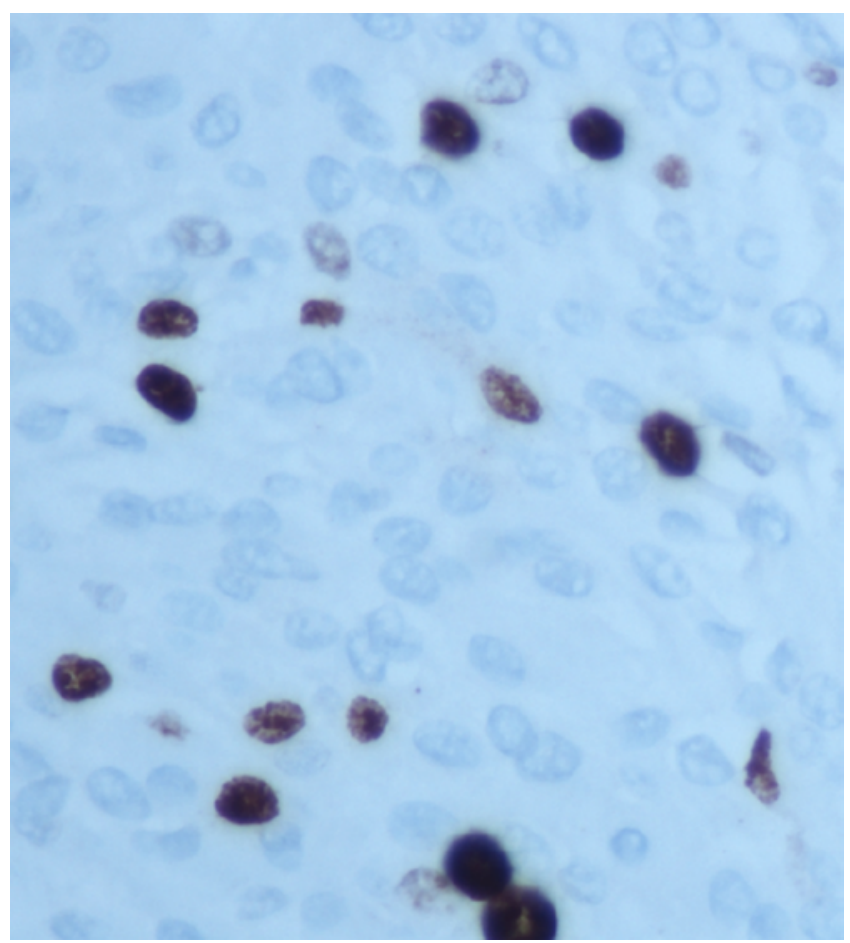

FIG. 2. Patient 1. Immunohistochemical staining showing a Ki-67 labeling index of $25 \%$, magnification $\times 40$.

\section{Patient 2 \\ Clinical Presentation and Operation}

A 73-year-old, right-handed woman presented with headaches, dizziness, left-sided hearing loss, left-sided facial numbness, confusion, and difficulty walking for 2 months. Examination revealed mild weakness in the left lower extremity (grade 4/5), left nose numbness, complete hearing loss on the left, and a Romberg sign. MRI revealed a $4.9 \times 4.2 \times 3.1-\mathrm{cm}$ left cerebellopontine angle tumor with mass effect upon the brainstem and fourth ventricle (Fig. $5 \mathrm{~A}$ ), consistent with a meningioma. She underwent a leftsided retrosigmoid craniotomy for diagnosis and planned subtotal resection (STR) of her tumor. Postoperative SRS was part of the initial management strategy.

\section{Pathological Findings}

Histopathological examination of the resected lesion revealed a meningioma with necrosis, calcifications, macronucleoli, and a high mitotic index (4-5 mitoses/10 hpf). These features classified the tumor as an atypical, WHO grade II meningioma. Immunostaining showed a high Ki67 labeling index at $10 \%-15 \%$.

\section{Postoperative Course}

The patient tolerated the procedure well with no complications. She was discharged to home on postoperative day 6. Her postoperative MRI demonstrated residual tumor, as known (Fig. 5B). Although the residual tumor volume was suitable for single-fraction SRS, our center was participating in an ongoing Radiation Therapy Oncology Group (RTOG, now NRG) study that mandated FRT to a dose of 60 Gy (Fig. 6). The patient was recovering as planned 


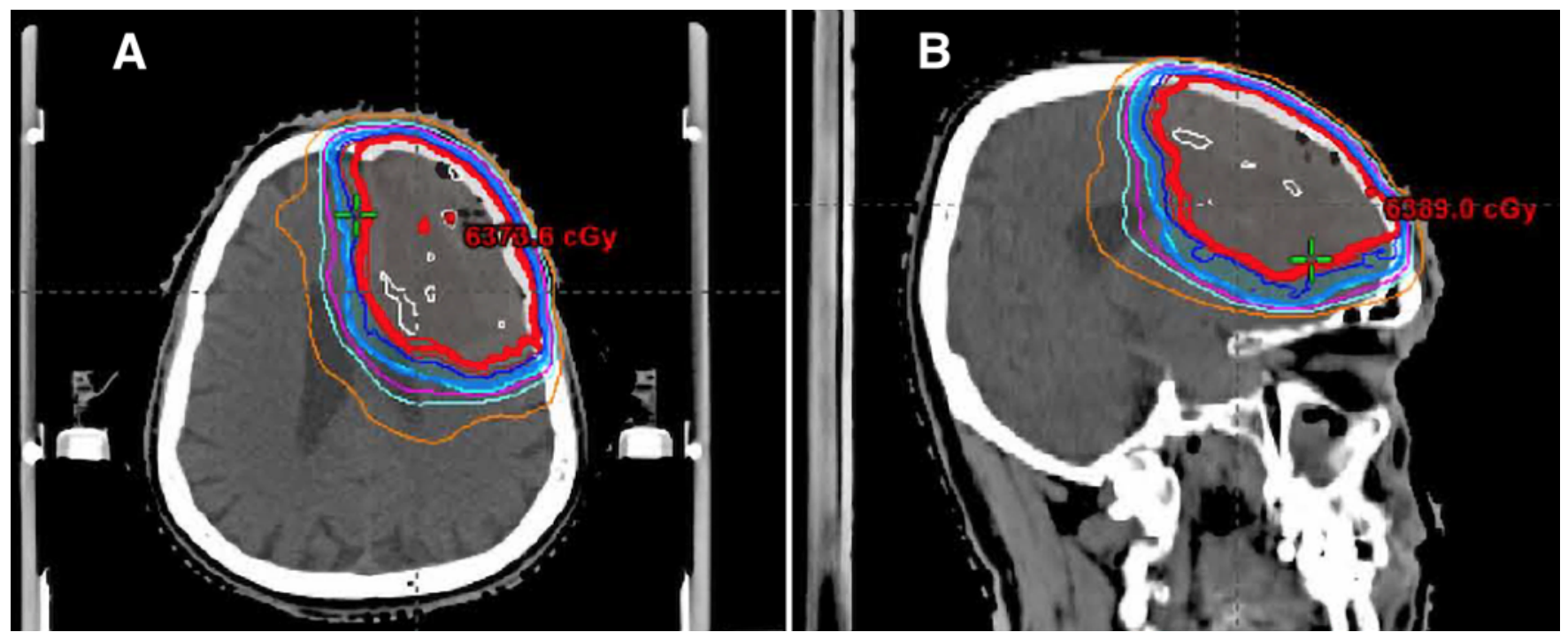

FIG. 3. RT treatment plan for patient 1. Cumulative dose-volume histogram, axial RT plan (A) and sagittal RT plan (B). The prescription dose of $60 \mathrm{~Gy}$ includes a large volume of the left frontal lobe adjacent to the resection cavity and encompasses the area of radiation necrosis shown

at her 6-month postoperative interval, and her MRI at that time showed a decrease in tumor volume compared to preRT size (Fig. 7A).

Twenty-one months after surgery, MRI showed an enhancing left temporal lobe lesion (Fig. 7B). Spectroscopy was inconclusive for radiation necrosis versus a neoplasm.
The patient now had memory and word finding difficulties. A stereotactic biopsy of the left temporal lesion was performed. Pathology was consistent with radiation necrosis, and the patient was started on low-dose steroid therapy. Her follow-up MRI 5 months later showed a stable enhancing lesion with decreased overall mass effect (Fig.
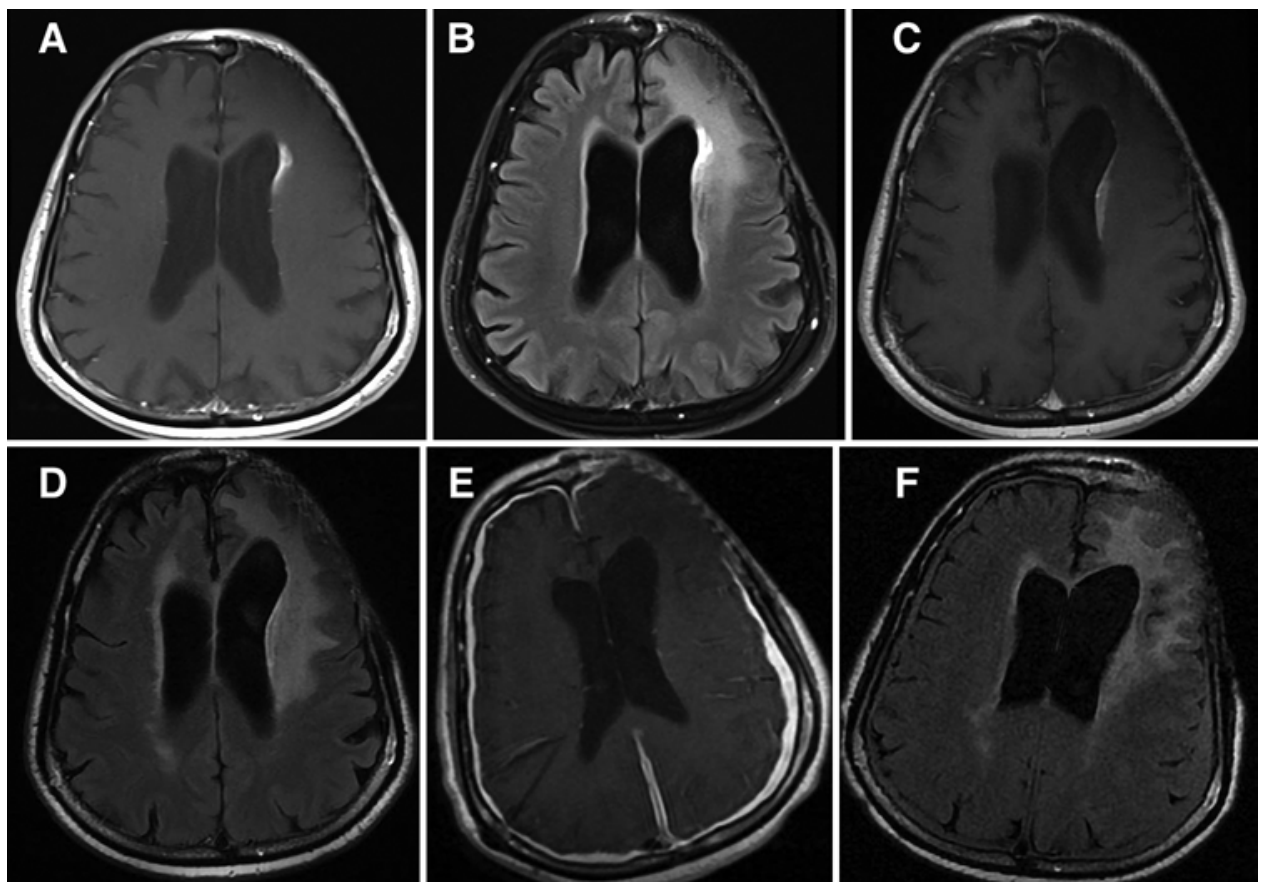

FIG. 4. Patient 1. Contrast-enhanced T1-weighted (A) and T2-weighted FLAIR (B) MR images 20 months postoperatively showing an increase in size of the enhancement along the frontal horn. Twenty-four-month postoperative T1-weighted contrast-enhanced (C) and T2-weighted FLAIR (D) MR images revealing left frontal periventricular enhancement, with ex vacuo dilatation of the left lateral ventricle and overall enlargement of the ventricles that is concerning for hydrocephalus. Fifty-two-month postoperative T1weighed contrast-enhanced (E) and T2-weighted FLAIR (F) MR images demonstrating resolution of the periventricular enhancement (note dural enhancement from ventriculoperitoneal shunt and the presence of a small subdural collection). 

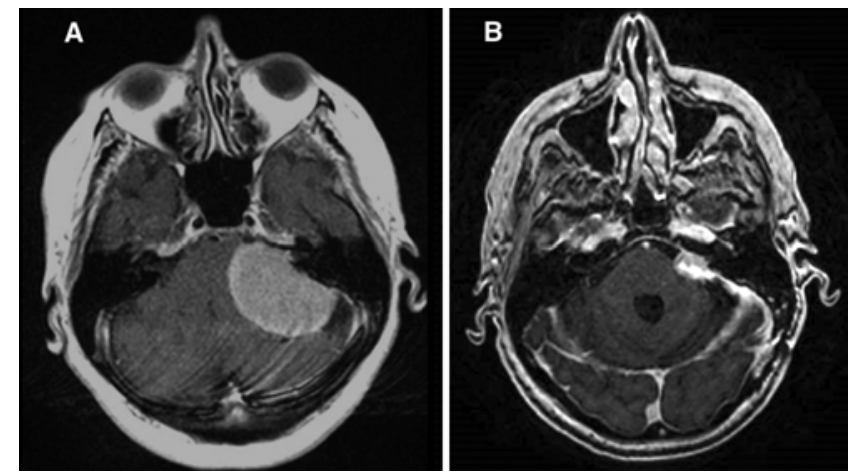

FIG. 5. Patient 2. A: Preoperative contrast-enhanced T1-weighted image showing a left cerebellopontine angle tumor with mass effect upon the brainstem and fourth ventricle. B: Two-month postoperative, pre-RT contrast-enhanced T1-weighted image demonstrating residual tumor.

7C). Her language and memory functions were improving. She was able to perform her ADL independently and continued to use a walker for assistance with ambulation.

Six months later, the patient was found down on the ground in her home. On examination, she had receptive aphasia and anterograde amnesia with slight right hemiparesis. MRI depicted an interval increase in the enhancing lesion extending into the left parietal lobe (Fig. 7D). Again, there was concern for a primary malignancy, with a sampling error on the prior biopsy; therefore, she underwent a stereotactic biopsy of the left parietal lesion. Histopathological evaluation revealed radiation necrosis, and she was started on prolonged course of steroid therapy. The patient never recovered to her immediate post-RT status and remained completely dependent for her ADL. Her last MRI, 38 months after RT, showed no evidence of meningioma growth.

\section{Discussion}

Patients with atypical meningiomas may have significant recurrence rates despite total or near-total surgical resection. The 5-year recurrence rates are as high as 50\% and $71 \%$ for Simpson grade I and grade I/II resection, respectively. ${ }^{2,19}$ Initial management options include observation, resection with or without adjuvant RT, SRS, or hypofractionated SRS versus irradiation alone for poor surgical candidates and inoperable tumors. ${ }^{27}$ Current guidelines recommend observation or adjuvant RT for patients who have had GTR of WHO grade II tumors. For patients who have had an STR, adjuvant RT is recommended..1 Adjuvant RT for patients with atypical meningiomas is associated with a median 5-year progression-free survival of $54.2 \%$ (range $38 \%-100 \%$ ) and OS of $67.5 \%$ (range, $51 \%-100 \%) .2,6,12,14,17,18,22,23,26,29,37$ The mitotic number and Ki-67 proliferative index have been evaluated as prognostic factors for prediction of recurrence for atypical meningiomas. ${ }^{5}$ With a mitotic number $<4$, local recurrence at 3 years was $34 \%$ in patients with a Ki-67 $>10 \%$ and $17.1 \%$ in those with a Ki-67 $\leq 10 \%$. For patients with a higher number of mitoses $(\geq 4)$, local recurrence at 3 years was found to be $48.6 \%$ and $31.1 \%$ for $\mathrm{Ki}-67>10 \%$ and $\leq 10 \%$, respectively. ${ }^{5}$

However, these studies are limited by their retrospective analyses. Therefore, the ROAM/EORTC-1308 trial was established to prospectively determine the role of adjuvant RT versus observation for patients with surgically resected atypical meningiomas. ${ }^{15}$

Patients with high-risk meningiomas-defined by RTOG 0539 as those having grade II recurrence, an STR of grade II, and any grade III meningiomas-have a 3-year overall survival of $78.6 \%$, local control of $68.9 \%$ and progression-free survival of $59.2 \%$ with adjuvant FRT. ${ }^{31}$ Intermediate-risk meningiomas, defined as either recurrent grade I or newly diagnosed GTR grade II, have a 3-year local failure rate of $4 \%$ with FRT. ${ }^{32}$

In this report, we highlight two patients who have had poor clinical outcomes following adjuvant treatment with FRT, despite having no evidence of disease recurrence. Both patients received postoperative FRT (Figs. 3 and 6).

\section{Treatment Volume and Dose}

Both patients received FRT using margins utilized
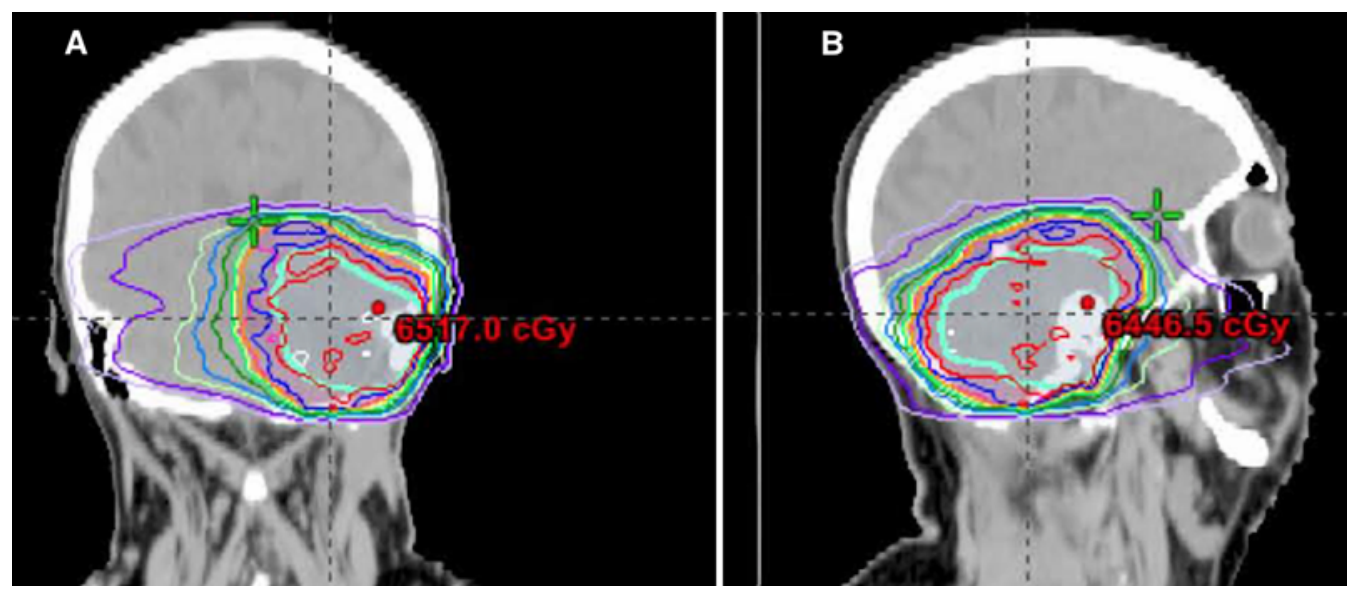

FIG. 6. RT treatment plan for patient 2. Cumulative dose-volume histogram, coronal RT plan (A) and sagittal RT plan (B). As in patient 1, the 60-Gy prescription dose covers a large volume of brain, including a significant part of the left temporal lobe. An SRS plan to the residual tumor would have avoided irradiating this large volume. 

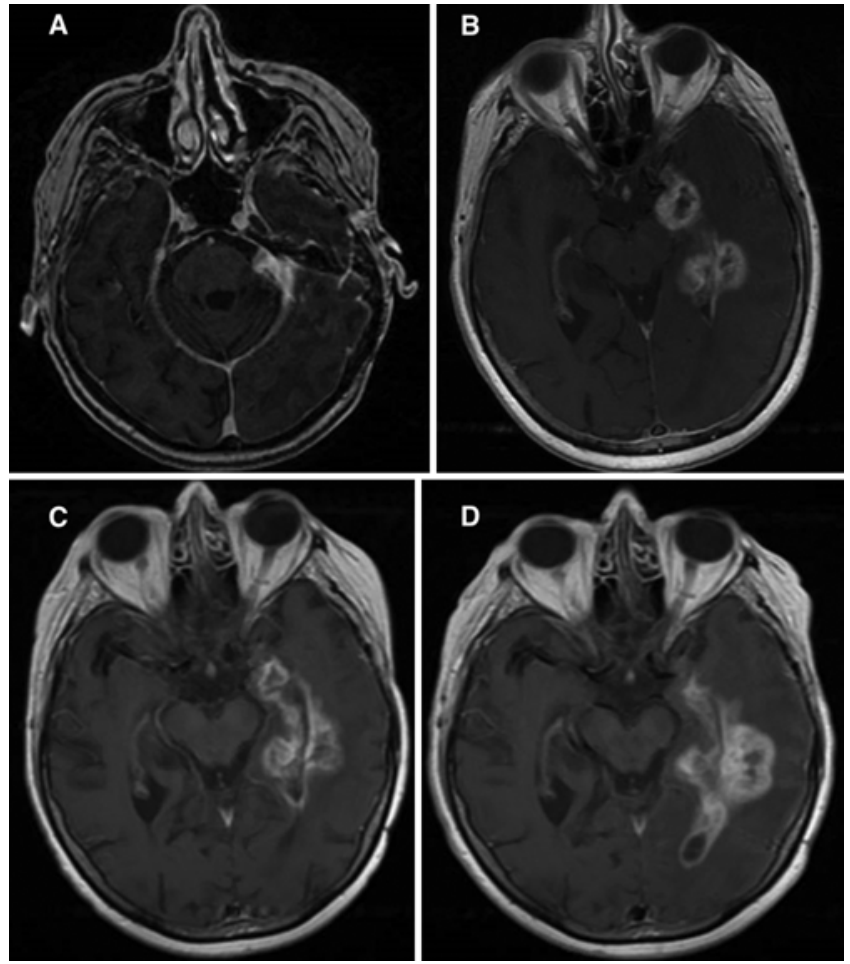

FIG. 7. Patient 2. A: Contrast-enhanced T1-weighted sequence MR image, 6 months after surgery and post-RT, showing decrease in the tumor volume compared to before RT. B: Twenty-one-month postoperative contrast-enhanced T1-weighted sequence depicting an enhancing left temporal lobe lesion that was biopsied. C: Five-month, post-biopsy, contrast-enhanced T1-weighted image revealing stable enhancing lesion with decreased overall mass effect. D: Contrast-enhanced T1-weighted MR image obtained 11 months after the first biopsy (time of second biopsy) showing an interval increase in the enhancing lesion extending into the left parietal lobe.

in RTOG 0539. First, a gross tumor volume (GTV) was defined by the tumor bed on postoperative MRI and included any residual nodular enhancement. Based on this GTV, two larger clinical tumor volumes (CTVs) were created to account for potential microscopic disease, including a CTV54, which was a $2-\mathrm{cm}$ expansion of the GTV to receive $54 \mathrm{~Gy}$ in 30 fractions, and a CTV60, which was a $1-\mathrm{cm}$ expansion of the GTV to receive 60 Gy in 30 fractions. The volumes treated were the planning tumor volumes (PTVs), PTV60 and PTV54, which were 5-mm expansions on the CTV60 and CTV54.

For the non-radiation oncologist, the volume that is included in the PTV can be surprising. The recommended PTV has ranged from a GTV plus a 2-cm margin, to a GTV plus $1 \mathrm{~cm}$, down to a GTV plus $2 \mathrm{~mm} .{ }^{32}$ In a previous report, Debus and colleagues used a head cast attached to a stereotactic frame to tightly focus the PTV for patients with meningiomas. Interestingly, with a median follow-up of 35 months, they have observed no margin failures. ${ }^{10}$

The rationale for fractionated treatment in these patients was based on literature that shows that patients who receive SRS for WHO grade II meningiomas experience high rates of recurrence outside the SRS volume. ${ }^{3,30}$ Huffmann et al. ${ }^{13}$ reviewed 15 patients treated with SRS to a median dose of 16 Gy. At 18-36 months, crude local control was $60 \%$. In 6 patients (40\%), disease progressed, one in-field, but all within the resection bed. Choi et al. ${ }^{9}$ also reviewed 25 patients with atypical meningioma, who had received a median SRS dose of $22 \mathrm{~Gy}$ in 1-4 fractions. Of the 9 recurrences seen, 3 were within the targeted region, 5 were elsewhere in the resection bed, and 1 was regional. These findings have suggested that the appropriate target volume for atypical meningioma may extend beyond the enhancing residual tumor and may include the entire resection bed. Future patterns-of-failure analyses are needed before definitive guidelines can be developed.

The optimal dose of FRT for atypical meningiomas also is currently being studied. The typical dose of $54 \mathrm{~Gy}$ for benign meningiomas may be insufficient for atypical and anaplastic primaries. A study by Hug et al. ${ }^{14}$ from Massachusetts General Hospital included 13 patients treated with surgery and proton-beam irradiation for malignant meningioma. Patients with cumulative doses exceeding 60 Gy (expressed as a cobalt Gray equivalent) manifested significantly better local control: 5-year actuarial local control was $100 \%$ for 6 patients treated with $\geq 60 \mathrm{~Gy}$, versus $0 \%$ for 7 patients receiving $<60$ Gy $(\mathrm{p}=0.025)$. Similarly, Boskos et al. ${ }^{6}$ detailed 24 patients with high-grade meningioma (79\% WHO Grade II), typically following STR, and saw a cause-specific survival at 5 years of $80 \%$ when the radiation dose was $>60$ Gy versus $24 \%$ when the dose was $\leq 60$ Gy $(\mathrm{p}=0.01)$, with a trend toward further improvement with doses $>65$ Gy $(p=0.06)$. RTOG 0539 treated intermediate-risk meningiomas with a dose of $60 \mathrm{~Gy}$, with promising 3-year data. We await the final results. ${ }^{32}$

\section{Treatment Toxicity}

RT toxicity rates for patients with atypical meningiomas range from $3.4 \%$ to $16.7 \%$. $2,6,12,14,17,22,23,26,29,37$ Specifically, cerebral necrosis rates from RT can be variable, with an incidence from as high as $23.1 \%^{33}$ (although this was for grade III tumors), $12.5 \%,{ }^{2}$ and $4.2 \%{ }^{6}$ and to one as low as $0.1 \% .{ }^{29}$ Grade 4 late toxicities, per Common Terminology Criteria for Adverse Events v3.0, have been reported for brain necrosis and cognitive disturbances ${ }^{29}$ The rate of such grade 4 and grade 3 toxicities was 2/119 (1.7\%) and $6 / 119(5.0 \%) .{ }^{29}$ Other toxicity rates include alopecia $(8.3 \%)$ and seizures $(4.2 \%){ }^{6}$ The risk of stroke after RT compared to SRS is 12-fold higher. ${ }^{25}$

Radiation necrosis is an important complication of SRS and FRT that should be recognized early and managed appropriately. Radiation changes on MRI include signal hypointensity on T1-weighted images and hyperintensity on T2-weighted images. CT scanning typically depicts radiation necrosis as hypodensity changes within the treatment field. Radiation necrosis is not a radiographic diagnosis alone. Rather, the onset of symptoms outside the postsurgical window and the absence of tumor recurrence with symptom onset and radiographic correlation together classify radiation necrosis. ${ }^{24}$

While acute complications are infrequent with RT, the cumulative dose of radiation can lead to significant neurocognitive changes or radiation necrosis. ${ }^{8}$ Hypofractionated SRS techniques have been described as causing less toxicity for larger tumor volumes. ${ }^{30}$ Bria et al. ${ }^{7}$ reported 
that hypofractionated SRS led to a $71 \%$ local control rate at 1 year in patients with grade II meningioma without any short-term toxicities. High-level evidence is still lacking with regard to direct comparisons of SRS, hypofractionated SRS, and RT in the adjuvant setting. ${ }^{36}$ Challenges arise with more invasive tumors, as SRS has a tighter conformal plan and could fall short in tumor control compared to RT. ${ }^{8}$ Comparatively, complication rates of SRS range from $2.5 \%$ to $37 \%$, while those for RT vary from $2 \%$ to $42 \%{ }^{4,20}$ Contributing factors include tumor volume, number of fractions, tumor histology, pretreatment edema, and cumulative radiation dose. The majority of post-SRS complications involve perilesional edema, and fractionation of SRS has led to decreased complication rates..$^{30}$ Adjuvant SRS has risks of tumor recurrence in the surgical cavity but can be used for focal tumor recurrence, and the risk of radiation necrosis from large-volume irradiation can thereby be avoided.

\section{Management of Toxicities}

Corticosteroid therapy is the treatment of choice for patients with treatment-related edema or radiation necrosis. Both of our patients had significant cognitive decline greater than 12 months after receiving RT, without evidence of tumor recurrence or progression. Steroid treatment helped at first in each case, but both patients continued to decline neurologically and sustain permanent deficits without any sign of tumor progression. Bevacizumab has been described as a treatment option for patients with steroid-resistant radiation necrosis. ${ }^{21,38}$ However, given that our patients did not have significant mass effect and were neurologically devastated, it was felt that there was no role for this therapy.

\section{Conclusions}

Neurosurgeons may refer patients with meningiomas for RT without an adequate appreciation of the differences in techniques that exist. Although SRS and FRT have revolutionized the management of brain tumors as minimally invasive and highly effective techniques, they are associated with inherent limitations and complications. We have described two patients who developed devastating complications after standard FRT. Treating smaller volumes with SRS, in one or several fractions, may decrease the risk of these life-altering events, but this approach may have increased risk of tumor recurrence in the resection cavity. The therapeutic profile of FRT may be further improved by reductions in the CTV and PTV margins traditionally employed. Comparative studies and an individualized approach to treatment are needed to achieve better tumor control and lower complication rates in patients with these complex tumors.

\section{References}

1. Achey RL, Gittleman H, Schroer J, Khanna V, Kruchko C, Barnholtz-Sloan JS: Non-malignant and malignant meningioma incidence and survival in the elderly from 2005-2015 using the Central Brain Tumor Registry of the United States. Neuro Oncol [epub ahead of print], 2018

2. Aghi MK, Carter BS, Cosgrove GR, Ojemann RG, Amin-
Hanjani S, Martuza RL, et al: Long-term recurrence rates of atypical meningiomas after gross total resection with or without postoperative adjuvant radiation. Neurosurgery 64:56-60, 2009

3. Attia A, Chan MD, Mott RT, Russell GB, Seif D, Daniel Bourland J, et al: Patterns of failure after treatment of atypical meningioma with gamma knife radiosurgery. J Neurooncol 108:179-185, 2012

4. Bagshaw HP, Burt LM, Jensen RL, Suneja G, Palmer CA, Couldwell WT, et al: Adjuvant radiotherapy for atypical meningiomas. J Neurosurg 126:1822-1828, 2017

5. Barrett OC, Hackney JR, McDonald AM, Willey CD, Bredel $\mathrm{M}$, Fiveash JB: Pathologic predictors of local recurrence in atypical meningiomas following gross total resection. Int J Radiat Oncol Biol Phys 103:453-459, 2019

6. Boskos C, Feuvret L, Noel G, Habrand JL, Pommier P, Alapetite $\mathrm{C}$, et al: Combined proton and photon conformal radiotherapy for intracranial atypical and malignant meningioma. Int J Radiat Oncol Biol Phys 75:399-406, 2009

7. Bria C, Wegner RE, Clump DA, Vargo JA, Mintz AH, Heron DE, et al: Fractionated stereotactic radiosurgery for the treatment of meningiomas. J Cancer Res Ther 7:52-57, 2011

8. Buerki RA, Horbinski CM, Kruser T, Horowitz PM, James CD, Lukas RV: An overview of meningiomas. Future Oncol 14:2161-2177, 2018

9. Choi CYH, Soltys SG, Gibbs IC, Harsh GR, Jackson PS, Lieberson RE, et al: Cyberknife stereotactic radiosurgery for treatment of atypical (WHO grade II) cranial meningiomas. Neurosurgery 67:1180-1188, 2010

10. Debus J, Wuendrich M, Pirzkall A, Hoess A, Schlegel W, Zuna I, et al: High efficacy of fractionated stereotactic radiotherapy of large base-of-skull meningiomas: long-term results. J Clin Oncol 19:3547-3553, 2001

11. Goldbrunner R, Minniti G, Preusser M, Jenkinson MD, Sallabanda K, Houdart E, et al: EANO guidelines for the diagnosis and treatment of meningiomas. Lancet Oncol 17:e383e391, 2016

12. Goyal LK, Suh JH, Mohan DS, Prayson RA, Lee J, Barnett GH: Local control and overall survival in atypical meningioma: a retrospective study. Int J Radiat Oncol Biol Phys 46:57-61, 2000

13. Huffmann BC, Reinacher PC, Gilsbach JM: Gamma knife surgery for atypical meningiomas. J Neurosurg 102 (Suppl):283-286, 2005

14. Hug EB, Devries A, Thornton AF, Munzenride JE, Pardo FS, Hedley-Whyte ET, et al: Management of atypical and malignant meningiomas: role of high-dose, 3D-conformal radiation therapy. J Neurooncol 48:151-160, 2000

15. Jenkinson MD, Javadpour M, Haylock BJ, Young B, Gillard H, Vinten J, et al: The ROAM/EORTC-1308 trial: Radiation versus Observation following surgical resection of Atypical Meningioma: study protocol for a randomised controlled trial. Trials 16:519, 2015

16. Jenkinson MD, Santarius T, Zadeh G, Aldape KD: Atypical meningioma-is it time to standardize surgical sampling techniques? Neuro Oncol 19:453-454, 2017

17. Kano H, Takahashi JA, Katsuki T, Araki N, Oya N, Hiraoka $\mathrm{M}$, et al: Stereotactic radiosurgery for atypical and anaplastic meningiomas. J Neurooncol 84:41-47, 2007

18. Kaur G, Sayegh ET, Larson A, Bloch O, Madden M, Sun MZ, et al: Adjuvant radiotherapy for atypical and malignant meningiomas: a systematic review. Neuro Oncol 16:628-636, 2014

19. Komotar RJ, Iorgulescu JB, Raper DMS, Holland EC, Beal $\mathrm{K}$, Bilsky $\mathrm{MH}$, et al: The role of radiotherapy following gross-total resection of atypical meningiomas. J Neurosurg 117:679-686, 2012

20. Kuhn EN, Taksler GB, Dayton O, Loganathan A, Bourland $\mathrm{D}$, Tatter SB, et al: Is there a tumor volume threshold for 
postradiosurgical symptoms? A single-institution analysis. Neurosurgery 75:536-545, 2014

21. Ma Y, Zheng C, Feng Y, Xu Q: Bevacizumab for the treatment of Gammaknife radiosurgery-induced brain radiation necrosis. J Craniofac Surg 28:e569-e571, 2017

22. Mahmood A, Qureshi NH, Malik GM: Intracranial meningiomas: analysis of recurrence after surgical treatment. Acta Neurochir (Wien) 126:53-58, 1994

23. Mair R, Morris K, Scott I, Carroll TA: Radiotherapy for atypical meningiomas. J Neurosurg 115:811-819, 2011

24. Mathiesen T, Kihlström L, Karlsson B, Lindquist C: Potential complications following radiotherapy for meningiomas. Surg Neurol 60:193-200, 2003

25. McClelland S III, Ciporen JN, Mitin T, Jaboin JJ: Long-term stroke risk of single-fraction photon-based stereotactic radiosurgery for meningioma. Clin Neurol Neurosurg 173:169172,2018

26. Milosevic MF, Frost PJ, Laperriere NJ, Wong CS, Simpson WJ: Radiotherapy for atypical or malignant intracranial meningioma. Int J Radiat Oncol Biol Phys 34:817-822, 1996

27. National Comprehensive Cancer Network: NCCN Guidelines: Central Nervous System Cancers. NCCN.org (http:// www.nccn.org/) [Accessed April 4, 2019]

28. Ostrom QT, Gittleman H, Xu J, Kromer C, Wolinsky Y, Kruchko C, et al: CBTRUS Statistical Report: Primary brain and other central nervous system tumors diagnosed in the United States in 2009-2013. Neuro Oncol 18 (suppl_5):v1v75, 2016

29. Pasquier D, Bijmolt S, Veninga T, Rezvoy N, Villa S, Krengli $\mathrm{M}$, et al: Atypical and malignant meningioma: outcome and prognostic factors in 119 irradiated patients. A multicenter, retrospective study of the Rare Cancer Network. Int J Radiat Oncol Biol Phys 71:1388-1393, 2008

30. Rogers L, Barani I, Chamberlain M, Kaley TJ, McDermott M, Raizer J, et al: Meningiomas: knowledge base, treatment outcomes, and uncertainties. A RANO review. J Neurosurg 122:4-23, 2015

31. Rogers L, Zhang P, Vogelbaum M, Perry A, Ashby LS, Modi J, et al: MNGI-08. High-risk meningioma: initial outcomes from NRG Oncology/RTOG-0539. Neuro Oncol 19 (Suppl 6): vi133, 2017

32. Rogers L, Zhang P, Vogelbaum MA, Perry A, Ashby LS, Modi JM, et al: Intermediate-risk meningioma: initial outcomes from NRG Oncology RTOG 0539. J Neurosurg 129:35-47, 2018
33. Rosenberg LA, Prayson RA, Lee J, Reddy C, Chao ST, Barnett GH, et al: Long-term experience with World Health Organization grade III (malignant) meningiomas at a single institution. Int J Radiat Oncol Biol Phys 74:427-432, 2009

34. Simpson D: The recurrence of intracranial meningiomas after surgical treatment. J Neurol Neurosurg Psychiatry 20:22-39, 1957

35. Stafford SL, Pollock BE, Foote RL, Link MJ, Gorman DA, Schomberg PJ, et al: Meningioma radiosurgery: tumor control, outcomes, and complications among 190 consecutive patients. Neurosurgery 49:1029-1038, 2001

36. Walcott BP, Nahed BV, Brastianos PK, Loeffler JS: Radiation treatment for WHO grade II and III meningiomas. Front Oncol 3:227, 2013

37. Yang SY, Park CK, Park SH, Kim DG, Chung YS, Jung HW: Atypical and anaplastic meningiomas: prognostic implications of clinicopathological features. J Neurol Neurosurg Psychiatry 79:574-580, 2008

38. Zhuang H, Yuan X, Yuan Z, Wang P: Indication of bevacizumab for cerebral radiation necrosis. Recent Patents Anticancer Drug Discov 12:272-277, 2017

\section{Disclosures}

The authors report no conflict of interest concerning the materials or methods used in this study or the findings specified in this paper.

\section{Author Contributions}

Conception and design: all authors. Acquisition of data: Rana. Analysis and interpretation of data: Dawley, Rana. Drafting the article: Dawley. Critically revising the article: all authors. Reviewed submitted version of manuscript: Dawley, Abou-AlShaar, Goenka, Schulder. Study supervision: Schulder.

\section{Correspondence}

Troy Dawley: Ascension Providence Hospitals, Michigan State University, College of Human Medicine, Southfield, MI. troy. dawley@ascension.org. 